Volume 70, Number 1, Pages 382-396 (2021)

DOI: $10.31801 /$ cfsuasmas. 645030

ISSN 1303-5991 E-ISSN 2618-6470

Received by the editors: November 11, 2019; Accepted: February 23, 2021

\title{
LUMP-TYPE SOLUTIONS OF A NEW EXTENDED $(3+1)$-DIMENSIONAL NONLINEAR EVOLUTION EQUATION
}

\author{
Yakup YILDIRIM ${ }^{1}$ and Emrullah YAŞAR ${ }^{2}$ \\ ${ }^{1}$ Department of Mathematics, Faculty of Arts and Sciences, \\ Near East University, 99138 Nicosia, CYPRUS \\ ${ }^{2}$ Department of Mathematics, Faculty of Arts and Sciences, \\ Uludag University, 16059 Bursa, TURKEY
}

\begin{abstract}
In this paper, we study lump-type solutions to a new extended $(3+1)$-dimensional nonlinear evolution equation which appears in the field of wave propagation in the nonlinear systems. We generate these types of solutions by considering the prime number $p=3$ of the generalized Hirota bilinear operators. With the help of Maple symbolic computations, we retrieve twentytwo classes of lump-type solutions which are a special kind of rational function solutions, localized in all directions in the space and describe various dispersive wave phenomena. These lump-type solutions are derived from positive quadratic function solutions by using the generalized Hirota bilinear form of the considered model. The lump solutions are recovered along with the existence conditions: Analyticity, positivity and localization in all directions. The required conditions of the analyticity and positivity of the solutions can be easily achieved by taking special choices of the involved parameters. The main ingredients for this scheme are to recover the Hirota bilinear forms and their generalized equivalences. Lastly, the graphical simulations of the exact solutions are depicted.
\end{abstract}

\section{INTRODUCTION}

Nonlinear evolution equations (NLEEs) model many physical phenomena in real world problems. NLEEs emerge in abundant scientific fields such as in fluid dynamics, nonlinear optical fibers, nuclear physics, electromagnetism, medicine, finance, mathematical biosciences, etc. To get a concrete picture to the physical phenomena through qualitative and quantitative features of NLEEs, it is imperatively important to investigate the analytic solutions of these equations. One of the important

2020 Mathematics Subject Classification. 35Q51, 37K40.

Keywords and phrases. Lump-type solution, generalized bilinear operator, a new extended $(3+1)$-dimensional nonlinear evolution equation, symbolic computation.

yakup.yildirim@neu.edu.tr-Corresponding author; eyasar@uludag.edu.tr

(D) 0000-0003-4443-3337; 0000-0003-4732-5753.

(C)2021 Ankara University Communications Faculty of Sciences University of Ankara-Series A1 Mathematics and Statistics 
results of these solutions is soliton solution. The soliton solutions of NLEEs [1, $2,3.4$ are special type traveling waves and localized exponentially in certain directions on plane or space. It is well known that the Hirota bilinear forms are main ingredients in constructing soliton solutions [5]. The soliton pulse implies a delicate balance between nonlinearity and dispersion effects [6]. Besides soliton solutions, there exist rational solutions to NLEEs. In contrast to soliton solutions, lump solutions are a special kind of rational function solutions, localized in all directions in the space. Lump solutions describe various dispersive wave phenomena and they can also be derived from taking long wave limits of soliton equations. Lump solutions are a kind of economical approximations to real physical waves, based on the computational time and the difficulty level. Different nonlinear terms can work together to formulate such solutions 25, 26] but there exist such solutions in linear model equations as well 27]. The important properties of the lump solutions are being that shapes, amplitudes, velocities of solitons after the collision with another soliton will keep and this is an elastic property of collision [28, 29,30,31, 32, 33. Many important techniques like Wronskian, Casoratian, Adomian decomposion method and bilinear Backlund transformations have been associated with rational function solutions 7 . Moreover, lump solutions of some NLEEs have been studied very recently $8: 9$ 10: 11 . Therefore, the investigation of lump solutions for NLEEs through Hirota bilineer forms is quite important and active task. The lump solution should satisfiy three important conditions: Analyticity, positivity and localization in all directions. In $(3+1)$ and $(4+1)$ dimension cases, the last condition -i.e localizationis not provided. They do not approach to zero in all directions due to the character of $(3+1)$ and $(4+1)$-dimensions in the resulting solutions, and thus, they are lumptype solutions but not lump solutions. We also note that rogue wave solutions are a special kind of lump or lump-type solutions, and are viewed in some research fields such as oceanography and nonlinear optics $7,12,13,14$.

One of the examples of NLEES is the $(3+1)$-dimensional nonlinear evolution equation (NLEE)

$$
3 u_{x z}-\left(2 u_{t}+u_{x x x}-2 u u_{x}\right)_{y}+2\left(u_{x} \partial_{x}^{-1} u_{y}\right)_{x}=0 .
$$

Eq. (1) has been studied by many researchers via different methods and many important results have been concluded. For instance, in [6] Wazwaz engendered multiple soliton and multiple singular soliton solutions to Eq. (1) by using the simplified form of the Hirota's methods. In 15, 16], authors constructed N-soliton solutions via the perturbation, Hirota bilinear, and the Wronskian determinant methods. Moreover, group invariant solutions of Eq. (1) are extracted by Lie's group theoretical approach in 17]. Eq. (1) was first appeared in [19] in the investigation of the algebraic-geometrical solutions. As demonstrated in 20,21, there exist a strong relationship between Eq. (1) and the Korteweg-de Vries(KdV) equation. This is due to the fact that Eq. (1) has the classical KdV equation

$$
v_{t}-6 v v_{x}+v_{x x x}=0
$$


as its main term $2 u_{t}+u_{x x x}-2 u u_{x}$ under the scale transformations 21]

$$
\begin{aligned}
u(x, t) & \rightarrow v\left(x^{\prime}, t^{\prime}\right), \\
x^{\prime} & \rightarrow \frac{1}{\sqrt{3}} x \\
t^{\prime} & \rightarrow \frac{1}{6 \sqrt{3}} t .
\end{aligned}
$$

Because of this fact, Eq. (1) may be modeled to study shallow-water waves and short waves in nonlinear dispersive models [21]. In this manuscript, we will deal with a new extended $(3+1)$-dimensional NLEE and present a general class of lumptype solutions 7] by Maple symbolic computation. The new extended $(3+1)$ dimensional NLEE has a Hirota bilinear form, and thereby, we will examine positive quadratic function solutions to the considered model. The obtained quadratic function solutions contain a set of free parameters, and taking special choices of the involved parameters we get a particular class of lump-type solutions. Finally, some concluding remarks and numerical simulations of the constructed lump-type solutions will be present at the end of the paper.

\section{An extended $(3+1)$-Dimensional NLEE}

A (3+1)-dimensional NLEE [6] is given by

$$
3 u_{x z}-2 u_{t y}-u_{3 x y}+4 u_{x} u_{y}+2 u u_{x y}+2 u_{x x} \partial_{x}^{-1}\left(u_{y}\right)=0
$$

where $\partial_{x}^{-1}$ is the inverse of $\partial_{x}$ with $\partial_{x} \partial_{x}^{-1}=\partial_{x}^{-1} \partial_{x}=I$, and

$$
\left(\partial_{x}^{-1} f\right)(x)=\int_{-\infty}^{x} f(t) d t
$$

With help of the dependent variable transformation $u(x, y, z, t)=-3[\ln f(x, y, z, t)]_{x x}$, the Hirota bilinear form is 15

$$
\begin{gathered}
\left(3 D_{x} D_{z}-2 D_{y} D_{t}-D_{x}^{3} D_{y}\right) f f= \\
6 f f_{x z}-6 f_{x} f_{z}-4 f f_{y t}+4 f_{y} f_{t}-2 f f_{3 x y}+2 f_{3 x} f_{y}+6 f_{x} f_{2 x y}-6 f_{2 x} f_{x y}=0
\end{gathered}
$$

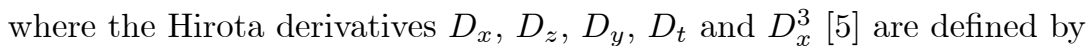

$$
D_{x}^{\alpha} D_{t}^{\beta}(f g)=\left.\left(\frac{\partial}{\partial x}-\frac{\partial}{\partial x^{\prime}}\right)^{\alpha}\left(\frac{\partial}{\partial t}-\frac{\partial}{\partial t^{\prime}}\right)^{\beta} f(x, t) g\left(x^{\prime}, t^{\prime}\right)\right|_{x=x^{\prime}, t=t^{\prime}} .
$$

The generalized differential operators for prime number $p$ have been introduced in $[18$ by $\mathrm{Ma}$ as the follows:

$$
D_{p, x}^{a} D_{p, t}^{b}(f g)=\left.\left(\frac{\partial}{\partial x}+\alpha_{p} \frac{\partial}{\partial x^{\prime}}\right)^{a}\left(\frac{\partial}{\partial t}+\alpha_{p} \frac{\partial}{\partial t^{\prime}}\right)^{b} f(x, t) g\left(x^{\prime}, t^{\prime}\right)\right|_{x=x^{\prime}, t=t^{\prime}}
$$




$$
=\left.\sum_{i=0}^{a} \sum_{j=0}^{b}\left(\begin{array}{l}
a \\
i
\end{array}\right)\left(\begin{array}{l}
b \\
j
\end{array}\right) \alpha_{p}^{i} \alpha_{p}^{j} \frac{\partial^{a-i}}{\partial x^{a-i}} \frac{\partial^{i}}{\partial x^{\prime(i)}} \frac{\partial^{b-j}}{\partial t^{b-j}} \frac{\partial^{j}}{\partial t^{\prime(j)}} f(x, t) g\left(x^{\prime}, t^{\prime}\right)\right|_{x=x^{\prime}, t=t^{\prime}}
$$

where

$$
\alpha_{p}^{s}=(-1)^{r_{p}(s)}, \quad s=r_{p}(s) \bmod p .
$$

We note that if $p=2 k(k \in \mathbb{N})$, all generalized bilinear differential operators (4) given above transform to Hirota bilinear operators, due to the fact that $D_{2 k, x}=D_{x}$ 5. For example if we take $p=3$ in (5), then we have

$$
\alpha_{3}=-1, \quad \alpha_{3}^{2}=\alpha_{3}^{3}=1, \quad \alpha_{3}^{4}=-1, \quad \alpha_{3}^{5}=\alpha_{3}^{6}=1, \ldots
$$

In what follows, we present the equivalent generalized Hitota bilinear derivatives of (3) corresponding to $p=3$

$$
\begin{gathered}
D_{3, x} D_{3, z} f f=2 f f_{x z}-2 f_{x} f_{z}, \\
D_{3, y} D_{3, t} f f=2 f f_{y t}-2 f_{y} f_{t}, \\
D_{3, x}^{3} D_{3, y} f f=6 f_{2 x} f_{x y} .
\end{gathered}
$$

We can correspondingly extend the Hirota bilinear equation (3), with $p=3$, into

$$
\left(3 D_{3, x} D_{3, z}-2 D_{3, y} D_{3, t}-D_{3, x}^{3} D_{3, y}\right) f f=6 f f_{x z}-6 f_{x} f_{z}-4 f f_{y t}+4 f_{y} f_{t}-6 f_{2 x} f_{x y}=0 .
$$

Based on the generalized bilinear form (6), we can show the extended $(3+1)$ dimensional NLEE under the transformation $u=-3(\ln f)_{x}$ as:

$$
\begin{gathered}
{\left[\frac{\left(3 D_{3, x} D_{3, z}-2 D_{3, y} D_{3, t}-D_{3, x}^{3} D_{3, y}\right) f f}{f^{2}}\right]_{x}=} \\
\left(\frac{4 u_{t}}{3}-\frac{u^{4}}{54}\right)_{y}-2\left(u_{z}+\frac{u_{x} u_{y}}{3}\right)_{x}+\frac{2}{9}\left(u u_{x}-\frac{u^{3}}{3}\right)_{x} \partial_{x}^{-1}\left(u_{y}\right)+\frac{2}{9} u^{2} u_{x y}+\frac{2 u u_{x} u_{y}}{3}=0 .
\end{gathered}
$$

We note that the extended $(3+1)$-dimensional NLEE (7) has more terms and higher order nonlinearities than the standard (3+1)-dimensional NLEE (2), and if $f$ solves the generalized bilinear equation (6), then $u=-3(\ln f)_{x}$ will present a solution to Eq. (7) which contains highly nonlinear terms than Eq.(2). It is readily seen that the generalized bilinear equation (6) is much simpler than the standard bilinear equation (3).

\section{Abundant lump-type SOlutions}

In order to gain lump-type solutions of Eq.(7), we assume $f$ in Eq. (6) is expressed in the following positive quadratic function form $[7,22,23,24,10$

$$
f=g^{2}+h^{2}+a_{11}
$$

with linear wave variables

$$
g=a_{1} x+a_{2} y+a_{3} z+a_{4} t+a_{5},
$$




$$
h=a_{6} x+a_{7} y+a_{8} z+a_{9} t+a_{10}
$$

where $a_{i}, 1 \leq i \leq 11$ are all real constants to be fixed later. Plugging Eq. (8) into Eq. (6) and putting the all the coefficients of different powers of $x, y, z, t$ to zero, we get a system of algebraic equations which contain $a_{i}(1 \leq i \leq 11)$ coefficients. After solving this messy system of algebraic equations with aid of Maple, we deduce the following results for $a_{i}(1 \leq i \leq 11)$.

\section{Result-1:}

$$
\begin{gathered}
a_{1}=\frac{3 a_{3} a_{4} a_{6}-2 a_{4}^{2} a_{7}+3 a_{6} a_{8} a_{9}-2 a_{7} a_{9}^{2}}{3\left(a_{3} a_{9}-a_{4} a_{8}\right)}, \\
a_{2}=\frac{3 a_{3}^{2} a_{6}-2 a_{3} a_{4} a_{7}+3 a_{6} a_{8}^{2}-2 a_{7} a_{8} a_{9}}{2\left(a_{3} a_{9}-a_{4} a_{8}\right)}, \\
a_{11}=\frac{\left(a_{4}^{2}+a_{9}^{2}\right)\left(a_{3} a_{4}+a_{8} a_{9}\right)\left(\begin{array}{c}
9 a_{3}^{2} a_{6}^{2}-12 a_{3} a_{4} a_{6} a_{7} \\
+4 a_{4}^{2} a_{7}^{2}+9 a_{6}^{2} a_{8}^{2} \\
-12 a_{6} a_{7} a_{8} a_{9}+4 a_{7}^{2} a_{9}^{2}
\end{array}\right)^{2}}{18\left(a_{3} a_{9}-a_{4} a_{8}\right)^{4}\left(3 a_{6} a_{8}-2 a_{7} a_{9}\right)} .
\end{gathered}
$$

Result-2:

$$
\begin{gathered}
a_{1}=\frac{2 a_{2}^{2} a_{9}-3 a_{2} a_{3} a_{6}-3 a_{6} a_{7} a_{8}+2 a_{7}^{2} a_{9}}{3\left(a_{2} a_{8}-a_{3} a_{7}\right)}, \\
a_{4}=\frac{2 a_{2} a_{3} a_{9}-3 a_{3}^{2} a_{6}-3 a_{6} a_{8}^{2}+2 a_{7} a_{8} a_{9}}{2\left(a_{2} a_{8}-a_{3} a_{7}\right)}, \\
a_{11}=\frac{\left(a_{2}^{2}+a_{7}^{2}\right)^{2}\left(2 a_{2} a_{9}-3 a_{3} a_{6}\right)\left(\begin{array}{c}
4 a_{2}^{2} a_{9}^{2}-12 a_{2} a_{3} a_{6} a_{9} \\
+9 a_{3}^{2} a_{6}^{2}+9 a_{6}^{2} a_{8}^{2} \\
-12 a_{6} a_{7} a_{8} a_{9}+4 a_{7}^{2} a_{9}^{2}
\end{array}\right)}{9\left(a_{2} a_{8}-a_{3} a_{7}\right)^{3}\left(3 a_{6} a_{8}-2 a_{7} a_{9}\right)} .
\end{gathered}
$$

\section{Result-3:}$$
a_{1}=\frac{2 a_{2} a_{4}^{2}+2 a_{2} a_{9}^{2}-3 a_{3} a_{6} a_{9}+3 a_{4} a_{6} a_{8}}{3\left(a_{3} a_{4}+a_{8} a_{9}\right)},
$$$$
a_{7}=-\frac{2 a_{2} a_{3} a_{9}-2 a_{2} a_{4} a_{8}-3 a_{3}^{2} a_{6}-3 a_{6} a_{8}^{2}}{2\left(a_{3} a_{4}+a_{8} a_{9}\right)},
$$$$
a_{11}=\frac{\left(a_{4}^{2}+a_{9}^{2}\right)\left(\begin{array}{l}
4 a_{2}^{2} a_{4}^{2}+4 a_{2}^{2} a_{9}^{2} \\
-12 a_{2} a_{3} a_{6} a_{9}+12 a_{2} a_{4} a_{6} a_{8} \\
+9 a_{3}^{2} a_{6}^{2}+9 a_{6}^{2} a_{8}^{2}
\end{array}\right)^{2}}{18\left(a_{3} a_{9}-a_{4} a_{8}\right)\left(a_{3} a_{4}+a_{8} a_{9}\right)^{2}\left(2 a_{2} a_{9}-3 a_{3} a_{6}\right)} .
$$

\section{Result-4:}

$$
\begin{gathered}
a_{2}=-\frac{a_{7}\left(3 a_{1}^{4} a_{6}+6 a_{1}^{2} a_{6}^{3}+3 a_{6}^{5}+2 a_{1}^{2} a_{11} a_{9}-2 a_{1} a_{11} a_{4} a_{6}\right)}{3 a_{1}^{5}+6 a_{1}^{3} a_{6}^{2}+3 a_{1} a_{6}^{4}-2 a_{1} a_{11} a_{6} a_{9}+2 a_{11} a_{4} a_{6}^{2}}, \\
a_{3}=-\frac{2 a_{7}\left(3 a_{1}^{4} a_{9}+6 a_{1}^{2} a_{6}^{2} a_{9}+3 a_{6}^{4} a_{9}+2 a_{1} a_{11} a_{4} a_{9}-2 a_{11} a_{4}^{2} a_{6}\right)}{3\left(3 a_{1}^{5}+6 a_{1}^{3} a_{6}^{2}+3 a_{1} a_{6}^{4}-2 a_{1} a_{11} a_{6} a_{9}+2 a_{11} a_{4} a_{6}^{2}\right)},
\end{gathered}
$$




$$
a_{8}=\frac{2 a_{7}\left(3 a_{1}^{4} a_{4}+6 a_{1}^{2} a_{6}^{2} a_{4}+3 a_{6}^{4} a_{4}-2 a_{1} a_{11} a_{9}^{2}+2 a_{11} a_{4} a_{6} a_{9}\right)}{3\left(3 a_{1}^{5}+6 a_{1}^{3} a_{6}^{2}+3 a_{1} a_{6}^{4}-2 a_{1} a_{11} a_{6} a_{9}+2 a_{11} a_{4} a_{6}^{2}\right)} .
$$

\section{Result-5:}

Result-6:

$$
\begin{gathered}
a_{2}=\frac{3 a_{3}\left(3 a_{1}^{4} a_{6}+6 a_{1}^{2} a_{6}^{3}+3 a_{6}^{5}+2 a_{1}^{2} a_{11} a_{9}-2 a_{1} a_{11} a_{4} a_{6}\right)}{2\left(3 a_{1}^{4} a_{9}+6 a_{1}^{2} a_{6}^{2} a_{9}+3 a_{6}^{4} a_{9}+2 a_{1} a_{11} a_{4} a_{9}-2 a_{11} a_{4}^{2} a_{6}\right)}, \\
a_{7}=-\frac{3 a_{3}\left(3 a_{1}^{5}+6 a_{1}^{3} a_{6}^{2}+3 a_{1} a_{6}^{4}-2 a_{1} a_{11} a_{6} a_{9}+2 a_{11} a_{4} a_{6}^{2}\right)}{2\left(3 a_{1}^{4} a_{9}+6 a_{1}^{2} a_{6}^{2} a_{9}+3 a_{6}^{4} a_{9}+2 a_{1} a_{11} a_{4} a_{9}-2 a_{11} a_{4}^{2} a_{6}\right)}, \\
a_{8}=-\frac{a_{3}\left(3 a_{1}^{4} a_{4}+6 a_{1}^{2} a_{6}^{2} a_{4}+3 a_{6}^{4} a_{4}-2 a_{1} a_{11} a_{9}^{2}+2 a_{11} a_{4} a_{6} a_{9}\right)}{3 a_{1}^{4} a_{9}+6 a_{1}^{2} a_{6}^{2} a_{9}+3 a_{6}^{4} a_{9}+2 a_{1} a_{11} a_{4} a_{9}-2 a_{11} a_{4}^{2} a_{6}} .
\end{gathered}
$$

$$
\begin{gathered}
a_{2}=\frac{3 a_{1}^{2} a_{3}+2 a_{1} a_{7} a_{9}+3 a_{3} a_{6}^{2}-2 a_{4} a_{6} a_{7}}{2\left(a_{1} a_{4}+a_{6} a_{9}\right)}, \\
a_{8}=\frac{3 a_{1} a_{3} a_{9}-3 a_{3} a_{4} a_{6}+2 a_{4}^{2} a_{7}+2 a_{7} a_{9}^{2}}{3\left(a_{1} a_{4}+a_{6} a_{9}\right)}, \\
a_{11}=\frac{3\left(a_{1}^{2}+a_{6}^{2}\right)^{2}\left(3 a_{1} a_{3}+2 a_{7} a_{9}\right)}{2\left(3 a_{3} a_{6}-2 a_{4} a_{7}\right)\left(a_{1} a_{9}-a_{4} a_{6}\right)} .
\end{gathered}
$$

\section{Result-7:}

$$
\begin{gathered}
a_{3}=\frac{1}{3 a_{11} a_{6}\left(a_{1} a_{7}-a_{2} a_{6}\right)}\left(3 a_{1}^{4} a_{2}^{2}+6 a_{1}^{3} a_{2} a_{6} a_{7}+3 a_{1}^{2} a_{2}^{2} a_{6}^{2}\right. \\
\left.+3 a_{1}^{2} a_{6}^{2} a_{7}^{2}+6 a_{1} a_{2} a_{6}^{3} a_{7}+3 a_{6}^{4} a_{7}^{2}+2 a_{1} a_{11} a_{2} a_{7} a_{9}-2 a_{11} a_{2}^{2} a_{6} a_{9}\right), \\
a_{4}=\frac{1}{2 a_{11} a_{6}\left(a_{1} a_{7}-a_{2} a_{6}\right)}\left(3 a_{1}^{5} a_{2}+3 a_{1}^{4} a_{6} a_{7}+6 a_{1}^{3} a_{2} a_{6}^{2}\right. \\
\left.+6 a_{1}^{2} a_{6}^{3} a_{7}+3 a_{1} a_{2} a_{6}^{4}+3 a_{6}^{5} a_{7}+2 a_{1}^{2} a_{11} a_{7} a_{9}-2 a_{1} a_{11} a_{2} a_{6} a_{9}\right), \\
a_{8}=\frac{3 a_{1}^{3} a_{2}+3 a_{1}^{2} a_{6} a_{7}+3 a_{1} a_{2} a_{6}^{2}+3 a_{6}^{3} a_{7}+2 a_{11} a_{7} a_{9}}{3 a_{11} a_{6}} .
\end{gathered}
$$

\section{Result-8:}

$$
\begin{gathered}
a_{3}=\frac{1}{a_{11} a_{7}\left(a_{1} a_{7}-a_{2} a_{6}\right)}\left(a_{1}^{3} a_{2}^{3}+a_{1}^{3} a_{2} a_{7}^{2}+a_{1}^{2} a_{2}^{2} a_{6} a_{7}+a_{1}^{2} a_{6} a_{7}^{3}\right. \\
\left.+a_{1} a_{2}^{3} a_{6}^{2}+a_{1} a_{2} a_{6}^{2} a_{7}^{2}+a_{2}^{2} a_{6}^{3} a_{7}+a_{6}^{3} a_{7}^{3}+a_{1} a_{11} a_{2} a_{7} a_{8}-a_{11} a_{2}^{2} a_{6} a_{8}\right), \\
a_{4}=\frac{3}{2 a_{11} a_{7}\left(a_{1} a_{7}-a_{2} a_{6}\right)}\left(a_{1}^{4} a_{2}^{2}+2 a_{1}^{3} a_{2} a_{6} a_{7}+a_{1}^{2} a_{2}^{2} a_{6}^{2}+a_{1}^{2} a_{6}^{2} a_{7}^{2}\right. \\
\left.+2 a_{1} a_{2} a_{6}^{3} a_{7}+a_{6}^{4} a_{7}^{2}+a_{1}^{2} a_{11} a_{7} a_{8}-a_{1} a_{11} a_{2} a_{6} a_{8}\right), \\
a_{9}=-\frac{3\left(a_{1}^{3} a_{2}+a_{1}^{2} a_{6} a_{7}+a_{1} a_{2} a_{6}^{2}+a_{6}^{3} a_{7}-a_{11} a_{6} a_{8}\right)}{2 a_{11} a_{7}}
\end{gathered}
$$

\section{Result-9:}

$$
a_{3}=\frac{2 a_{2}\left(3 a_{1}^{4} a_{9}+6 a_{1}^{2} a_{6}^{2} a_{9}+3 a_{6}^{4} a_{9}+2 a_{1} a_{11} a_{4} a_{9}-2 a_{11} a_{4}^{2} a_{6}\right)}{3\left(3 a_{1}^{4} a_{6}+6 a_{1}^{2} a_{6}^{3}+3 a_{6}^{5}+2 a_{1}^{2} a_{11} a_{9}-2 a_{1} a_{11} a_{4} a_{6}\right)},
$$




$$
\begin{gathered}
a_{7}=-\frac{a_{2}\left(3 a_{1}^{5}+6 a_{1}^{3} a_{6}^{2}+3 a_{1} a_{6}^{4}-2 a_{1} a_{11} a_{6} a_{9}+2 a_{11} a_{4} a_{6}^{2}\right)}{3 a_{1}^{4} a_{6}+6 a_{1}^{2} a_{6}^{3}+3 a_{6}^{5}+2 a_{1}^{2} a_{11} a_{9}-2 a_{1} a_{11} a_{4} a_{6}}, \\
a_{8}=-\frac{2 a_{2}\left(3 a_{1}^{4} a_{4}+6 a_{1}^{2} a_{6}^{2} a_{4}+3 a_{6}^{4} a_{4}-2 a_{1} a_{11} a_{9}^{2}+2 a_{11} a_{4} a_{6} a_{9}\right)}{3\left(3 a_{1}^{4} a_{6}+6 a_{1}^{2} a_{6}^{3}+3 a_{6}^{5}+2 a_{1}^{2} a_{11} a_{9}-2 a_{1} a_{11} a_{4} a_{6}\right)} .
\end{gathered}
$$

\section{Result-10:}

$$
\begin{gathered}
a_{3}=-\frac{3 a_{1} a_{8} a_{9}-2 a_{2} a_{4}^{2}-2 a_{2} a_{9}^{2}-3 a_{4} a_{6} a_{8}}{3\left(a_{1} a_{4}+a_{6} a_{9}\right)}, \\
a_{7}=\frac{3 a_{1}^{2} a_{8}-2 a_{1} a_{2} a_{9}+2 a_{2} a_{4} a_{6}+2 a_{6}^{2} a_{8}}{2\left(a_{1} a_{4}+a_{6} a_{9}\right)}, \\
a_{11}=-\frac{3\left(a_{1}^{2}+a_{6}^{2}\right)^{2}\left(2 a_{2} a_{4}+3 a_{6} a_{8}\right)}{2\left(a_{1} a_{9}-a_{4} a_{6}\right)\left(3 a_{1} a_{8}-2 a_{2} a_{9}\right)} .
\end{gathered}
$$

\section{Result-11:}

$$
\begin{gathered}
a_{3}=\frac{2\left(a_{1} a_{2} a_{4}-a_{1} a_{7} a_{9}+a_{2} a_{6} a_{9}+a_{4} a_{6} a_{7}\right)}{3\left(a_{1}^{2}+a_{6}^{2}\right)}, \\
a_{8}=\frac{2\left(a_{1} a_{2} a_{9}+a_{1} a_{4} a_{7}-a_{2} a_{4} a_{6}+a_{6} a_{7} a_{9}\right)}{3\left(a_{1}^{2}+a_{6}^{2}\right)}, \\
a_{11}=-\frac{3\left(a_{1}^{2}+a_{6}^{2}\right)^{2}\left(a_{1} a_{2}+a_{6} a_{7}\right)}{2\left(a_{1} a_{9}-a_{4} a_{6}\right)\left(a_{1} a_{7}-a_{2} a_{6}\right)} .
\end{gathered}
$$

\section{Result-12:}

$$
\begin{gathered}
a_{4}=\frac{3 a_{1} a_{3}^{2}+3 a_{1} a_{8}^{2}-2 a_{2} a_{8} a_{9}+2 a_{3} a_{7} a_{9}}{2\left(a_{2} a_{3}+a_{7} a_{8}\right)} \\
a_{6}=-\frac{3 a_{1} a_{2} a_{8}-3 a_{1} a_{3} a_{7}-2 a_{2}^{2} a_{9}-2 a_{7}^{2} a_{9}}{3\left(a_{2} a_{3}+a_{7} a_{8}\right)} \\
a_{11}=-\frac{\left(\begin{array}{l}
9 a_{1}^{2} a_{3}^{2}+9 a_{1}^{2} a_{8}^{2} \\
-12 a_{1} a_{2} a_{8} a_{9} \\
+12 a_{1} a_{3} a_{7} a_{9} \\
+4 a_{2}^{2} a_{9}^{2}+4 a_{7}^{2} a_{9}^{2}
\end{array}\right)}{9\left(a_{2} a_{8}^{2}-a_{3} a_{7}\right)\left(3 a_{1} a_{3}+2 a_{7} a_{3}+a_{7} a_{8}\right)^{2}\left(3 a_{1} a_{8}-2 a_{2} a_{9}\right)} .
\end{gathered}
$$

\section{Result-13:}$$
a_{4}=-\frac{3\left(a_{1}^{3} a_{2}+a_{1}^{2} a_{6} a_{7}+a_{1} a_{2} a_{6}^{2}+a_{6}^{3} a_{7}-a_{1} a_{11} a_{3}\right)}{2 a_{11} a_{2}},
$$$$
a_{8}=-\frac{1}{a_{11} a_{2}\left(a_{1} a_{7}-a_{2} a_{6}\right)}\left(a_{1}^{3} a_{2}^{3}+a_{1}^{3} a_{2} a_{7}^{2}+a_{1}^{2} a_{2}^{2} a_{6} a_{7}\right.
$$$$
+a_{1}^{2} a_{6} a_{7}^{3}+a_{1} a_{2}^{3} a_{6}^{2}+a_{1} a_{2} a_{6}^{2} a_{7}^{2}+a_{2}^{2} a_{6}^{3} a_{7}+a_{6}^{3} a_{7}^{3}
$$$$
\left.-a_{1} a_{11} a_{3} a_{7}^{2}+a_{11} a_{2} a_{3} a_{6} a_{7}\right),
$$$$
a_{9}=-\frac{3}{2 a_{11} a_{2}\left(a_{1} a_{7}-a_{2} a_{6}\right)}\left(a_{1}^{4} a_{2}^{2}+2 a_{1}^{3} a_{2} a_{6} a_{7}\right.
$$$$
+a_{1}^{2} a_{2}^{2} a_{6}^{2}+a_{1}^{2} a_{6}^{2} a_{7}^{2}+2 a_{1} a_{2} a_{6}^{3} a_{7}+a_{6}^{4} a_{7}^{2}
$$ 


\section{Result-14:}

$$
\left.-a_{1} a_{11} a_{3} a_{6} a_{7}+a_{11} a_{2} a_{3} a_{6}^{2}\right) .
$$

\section{Result-15:}

$$
\begin{gathered}
a_{4}=\frac{3 a_{1}^{2} a_{3}+2 a_{1} a_{7} a_{9}-2 a_{2} a_{6} a_{9}+3 a_{3} a_{6}^{2}}{2\left(a_{1} a_{2}+a_{6} a_{7}\right)}, \\
a_{8}=\frac{3 a_{1} a_{3} a_{7}+2 a_{2}^{2} a_{9}-3 a_{2} a_{3} a_{6}+2 a_{7}^{2} a_{9}}{3\left(a_{1} a_{2}+a_{6} a_{7}\right)}, \\
a_{11}=-\frac{3\left(a_{1}^{2}+a_{6}^{2}\right)\left(a_{1} a_{2}+a_{6} a_{7}\right)^{2}}{\left(2 a_{2} a_{9}-3 a_{3} a_{6}\right)\left(a_{1} a_{7}-a_{2} a_{6}\right)} .
\end{gathered}
$$

$$
\begin{gathered}
a_{6}=-\frac{3 a_{1} a_{3} a_{4}+3 a_{1} a_{8} a_{9}-2 a_{2} a_{4}^{2}-2 a_{2} a_{9}^{2}}{3\left(a_{3} a_{9}-a_{4} a_{8}\right)}, \\
a_{7}=-\frac{3 a_{1} a_{3}^{2}+3 a_{1} a_{8}^{2}-2 a_{2} a_{3} a_{4}-2 a_{2} a_{8} a_{9}}{2\left(a_{3} a_{9}-a_{4} a_{8}\right)}, \\
a_{11}=\frac{\left(\begin{array}{c}
9 a_{1}^{2} a_{3}^{2}+9 a_{1}^{2} a_{8}^{2} \\
-12 a_{1} a_{2} a_{3} a_{4} \\
-12 a_{1} a_{2} a_{8} a_{9} \\
+4 a_{2}^{2} a_{4}^{2}+4 a_{2}^{2} a_{9}^{2}
\end{array}\right)^{2}}{18\left(a_{3}^{2} a_{9}-a_{4} a_{8}\right)^{4}\left(3 a_{1} a_{3}-2 a_{2} a_{4}\right)} .
\end{gathered}
$$

Result-16:

$$
\begin{aligned}
& a_{6}=\frac{3 a_{1} a_{2} a_{3}+3 a_{1} a_{7} a_{8}-2 a_{2}^{2} a_{4}-2 a_{4} a_{7}^{2}}{3\left(a_{2} a_{8}-a_{3} a_{7}\right)}, \\
& a_{9}=\frac{3 a_{1} a_{3}^{2}+3 a_{1} a_{8}^{2}-2 a_{2} a_{3} a_{4}-2 a_{4} a_{7} a_{8}}{2\left(a_{2} a_{8}-a_{3} a_{7}\right)}, \\
& \frac{\left(a_{2}^{2}+a_{7}^{2}\right)^{2}\left(3 a_{1} a_{8}-2 a_{4} a_{7}\right)\left(\begin{array}{l}
9 a_{1}^{2} a_{3}^{2}+9 a_{1}^{2} a_{8}^{2} \\
-12 a_{1} a_{2} a_{3} a_{4} \\
-12 a_{1} a_{4} a_{7} a_{8} \\
+4 a_{2}^{2} a_{4}^{2}+4 a_{4}^{2} a_{7}^{2}
\end{array}\right)}{9\left(a_{2} a_{8}-a_{3} a_{7}\right)^{3}\left(3 a_{1} a_{3}-2 a_{2} a_{4}\right)} .
\end{aligned}
$$

\section{Result-17:}

$$
\begin{gathered}
a_{7}=-\frac{3 a_{1}^{2} a_{3}-2 a_{1} a_{2} a_{4}-2 a_{2} a_{6} a_{9}+3 a_{3} a_{6}^{2}}{2\left(a_{1} a_{9}-a_{4} a_{6}\right)}, \\
a_{8}=-\frac{3 a_{1} a_{3} a_{4}-2 a_{2} a_{4}^{2}-2 a_{2} a_{9}^{2}+3 a_{3} a_{6} a_{9}}{3\left(a_{1} a_{9}-a_{4} a_{6}\right)}, \\
a_{11}=\frac{3\left(a_{1}^{2}+a_{6}^{2}\right)^{2}\left(2 a_{2} a_{9}-3 a_{3} a_{6}\right)}{2\left(a_{1} a_{9}-a_{4} a_{6}\right)\left(3 a_{1} a_{3}-2 a_{2} a_{4}\right)} .
\end{gathered}
$$

\section{Result-18:}

$$
a_{8}=-\frac{3 a_{1} a_{2} a_{3}-2 a_{2}^{2} a_{4}+3 a_{3} a_{6} a_{7}-2 a_{4} a_{7}^{2}}{3\left(a_{1} a_{7}-a_{2} a_{6}\right)},
$$


Result-19:

$$
\begin{gathered}
a_{9}=-\frac{3 a_{1}^{2} a_{3}-2 a_{1} a_{2} a_{4}+3 a_{3} a_{6}^{2}-2 a_{4} a_{6} a_{7}}{2\left(a_{1} a_{7}-a_{2} a_{6}\right)}, \\
a_{11}=\frac{3\left(a_{1}^{2}+a_{6}^{2}\right)\left(a_{1} a_{2}+a_{6} a_{7}\right)}{3 a_{1} a_{3}-2 a_{2} a_{4}} .
\end{gathered}
$$

$$
\begin{gathered}
a_{7}=-\frac{3 a_{1}^{3} a_{2}+3 a_{1} a_{2} a_{6}^{2}-3 a_{1} a_{11} a_{3}+2 a_{11} a_{2} a_{4}}{3 a_{6}\left(a_{1}^{2}+a_{6}^{2}\right)} \\
a_{9}=\frac{a_{6}\left(9 a_{1}^{4} a_{3}+18 a_{1}^{2} a_{3} a_{6}^{2}+9 a_{3} a_{6}^{4}-6 a_{1} a_{11} a_{3} a_{4}+4 a_{11} a_{2} a_{4}^{2}\right)}{2\left(3 a_{1}^{4} a_{2}+6 a_{1}^{2} a_{2} a_{6}^{2}+3 a_{2} a_{6}^{4}-3 a_{1}^{2} a_{11} a_{3}+2 a_{1} a_{11} a_{2} a_{4}\right)} \\
a_{8}=-\frac{1}{9 a_{6}\left(a_{1}^{2}+a_{6}^{2}\right)\left(\begin{array}{l}
3 a_{1}^{4} a_{2}+6 a_{1}^{2} a_{2} a_{6}^{2} \\
+3 a_{2} a_{6}^{4}-3 a_{1}^{2} a_{11} a_{3} \\
+2 a_{1} a_{11} a_{2} a_{4}
\end{array}\right)} \\
\times\left(18 a_{1}^{6} a_{2}^{2} a_{4}+54 a_{1}^{4} a_{2}^{2} a_{4} a_{6}^{2}+54 a_{1}^{2} a_{2}^{2} a_{4} a_{6}^{4}+18 a_{2}^{2} a_{4} a_{6}^{6}\right. \\
-36 a_{1}^{4} a_{11} a_{2} a_{3} a_{4}+24 a_{1}^{3} a_{11} a_{2}^{2} a_{4}^{2}-27 a_{1}^{3} a_{11} a_{3}^{2} a_{6}^{2}-18 a_{1}^{2} a_{11} a_{2} a_{3} a_{4} a_{6}^{2} \\
+24 a_{1} a_{11} a_{2}^{2} a_{4}^{2} a_{6}^{2}-27 a_{1} a_{11} a_{3}^{2} a_{6}^{4}+18 a_{11} a_{2} a_{3} a_{4} a_{6}^{4} \\
\left.+18 a_{1}^{2} a_{11}^{2} a_{3}^{2} a_{4}-24 a_{1} a_{11}^{2} a_{2} a_{3} a_{4}^{2}+8 a_{11}^{2} a_{2}^{2} a_{4}^{3}\right)
\end{gathered}
$$

\section{Result-20:}

$$
\begin{gathered}
a_{4}=-\frac{9 a_{1}^{4} a_{6} a_{8}+18 a_{1}^{2} a_{6}^{3} a_{8}+9 a_{6}^{5} a_{8}+6 a_{1}^{2} a_{11} a_{8} a_{9}-4 a_{1} a_{11} a_{2} a_{9}^{2}}{2\left(3 a_{1}^{4} a_{2}+6 a_{1}^{2} a_{2} a_{6}^{2}+3 a_{2} a_{6}^{4}-3 a_{1} a_{11} a_{6} a_{8}+2 a_{11} a_{2} a_{6} a_{9}\right)}, \\
a_{7}=-\frac{3\left(a_{1}^{3} a_{2}+a_{1} a_{2} a_{6}^{2}-a_{11} a_{6} a_{8}\right)}{3 a_{1}^{2} a_{6}+3 a_{6}^{3}+2 a_{11} a_{9}}, \\
a_{3}=\frac{1}{3\left(3 a_{1}^{2} a_{6}+3 a_{6}^{3}+2 a_{11} a_{9}\right)\left(\begin{array}{l}
3 a_{1}^{4} a_{2}+6 a_{1}^{2} a_{2} a_{6}^{2} \\
+3 a_{2} a_{6}^{4}-3 a_{1} a_{11} a_{6} a_{8} \\
+2 a_{11} a_{2} a_{6} a_{9}
\end{array}\right)} \\
\times\left(18 a_{1}^{6} a_{2}^{2} a_{9}+54 a_{1}^{4} a_{2}^{2} a_{6}^{2} a_{9}+54 a_{1}^{2} a_{2}^{2} a_{6}^{4} a_{9}+18 a_{2}^{2} a_{6}^{6} a_{9}-54 a_{1}^{3} a_{11} a_{2} a_{6} a_{8} a_{9}\right. \\
+24 a_{1}^{2} a_{11} a_{2}^{2} a_{6} a_{9}^{2}-27 a_{1}^{2} a_{11} a_{6}^{3} a_{8}^{2}-54 a_{1} a_{11} a_{2} a_{6}^{3} a_{8} a_{9}+24 a_{11} a_{2}^{2} a_{6}^{3} a_{9}^{2} \\
\left.-27 a_{11} a_{6}^{5} a_{8}^{2}-12 a_{1} a_{11}^{2} a_{2} a_{8} a_{9}^{2}+8 a_{11}^{2} a_{2}^{2} a_{9}^{3}\right) .
\end{gathered}
$$

\section{Result-21:}

$$
\begin{gathered}
a_{2}=-\frac{3\left(a_{1}^{2} a_{6} a_{7}+a_{6}^{3} a_{7}-a_{1} a_{11} a_{3}\right)}{3 a_{1}^{3}+3 a_{1} a_{6}^{2}+2 a_{11} a_{4}}, \\
a_{9}=-\frac{9 a_{1}^{5} a_{3}+18 a_{1}^{3} a_{3} a_{6}^{2}+9 a_{1} a_{3} a_{6}^{4}+6 a_{11} a_{3} a_{4} a_{6}^{2}-4 a_{11} a_{4}^{2} a_{6} a_{7}}{2\left(3 a_{1}^{4} a_{7}+6 a_{1}^{2} a_{6}^{2} a_{7}+3 a_{6}^{4} a_{7}-3 a_{1} a_{11} a_{3} a_{6}+2 a_{1} a_{11} a_{4} a_{7}\right)}, \\
a_{8}=\frac{1}{3\left(3 a_{1}^{3}+3 a_{1} a_{6}^{2}+2 a_{11} a_{4}\right)\left(\begin{array}{l}
3 a_{1}^{4} a_{7}+6 a_{1}^{2} a_{6}^{2} a_{7} \\
+3 a_{6}^{4} a_{7}-3 a_{1} a_{11} a_{3} a_{6} \\
+2 a_{1} a_{11} a_{4} a_{7}
\end{array}\right)},
\end{gathered}
$$




$$
\begin{gathered}
\times\left(18 a_{1}^{6} a_{4} a_{7}^{2}+54 a_{1}^{4} a_{4} a_{6}^{2} a_{7}^{2}+54 a_{1}^{2} a_{4} a_{6}^{4} a_{7}^{2}+18 a_{4} a_{6}^{6} a_{7}^{2}-27 a_{1}^{5} a_{11} a_{3}^{2}\right. \\
-27 a_{1}^{3} a_{11} a_{3}^{2} a_{6}^{2}-54 a_{1}^{3} a_{11} a_{3} a_{4} a_{6} a_{7}+24 a_{1}^{3} a_{11} a_{4}^{2} a_{7}^{2}-54 a_{1} a_{11} a_{3} a_{4} a_{6}^{3} a_{7} \\
\left.+24 a_{1} a_{11} a_{4}^{2} a_{6}^{2} a_{7}^{2}-12 a_{11}^{2} a_{3} a_{4}^{2} a_{6} a_{7}+8 a_{11}^{2} a_{4}^{3} a_{7}^{2}\right) .
\end{gathered}
$$

\section{Result-22:}

$$
\begin{gathered}
a_{2}=-\frac{3 a_{1}^{2} a_{6} a_{7}+3 a_{6}^{3} a_{7}-3 a_{11} a_{6} a_{8}+2 a_{11} a_{7} a_{9}}{3 a_{1}\left(a_{1}^{2}+a_{6}^{2}\right)} \\
a_{4}=\frac{a_{1}\left(9 a_{1}^{4} a_{8}+18 a_{1}^{2} a_{6}^{2} a_{8}+9 a_{6}^{4} a_{8}-6 a_{11} a_{6} a_{8} a_{9}+4 a_{11} a_{7} a_{9}^{2}\right)}{2\left(3 a_{1}^{4} a_{7}+6 a_{1}^{2} a_{6}^{2} a_{7}+3 a_{6}^{4} a_{7}-3 a_{11} a_{6}^{2} a_{8}+2 a_{11} a_{6} a_{7} a_{9}\right)}, \\
a_{3}=-\frac{1}{9 a_{1}\left(a_{1}^{2}+a_{6}^{2}\right)\left(\begin{array}{l}
3 a_{1}^{4} a_{7}+6 a_{1}^{2} a_{6}^{2} a_{7} \\
+3 a_{6}^{4} a_{7}-3 a_{11} a_{6}^{2} a_{8} \\
+2 a_{11} a_{6} a_{7} a_{9}
\end{array}\right)} \\
\times\left(18 a_{1}^{6} a_{7}^{2} a_{9}+54 a_{1}^{4} a_{6}^{2} a_{7}^{2} a_{9}+54 a_{1}^{2} a_{6}^{4} a_{7}^{2} a_{9}+18 a_{6}^{6} a_{7}^{2} a_{9}\right. \\
-27 a_{1}^{4} a_{11} a_{6} a_{8}^{2}+18 a_{1}^{4} a_{11} a_{7} a_{8} a_{9}-27 a_{1}^{2} a_{11} a_{6}^{3} a_{8}^{2} \\
-18 a_{1}^{2} a_{11} a_{6}^{2} a_{7} a_{8} a_{9}+24 a_{1}^{2} a_{11} a_{6} a_{7}^{2} a_{9}^{2}-36 a_{11} a_{6}^{4} a_{7} a_{8} a_{9} \\
\left.+24 a_{11} a_{6}^{3} a_{7}^{2} a_{9}^{2}+18 a_{11}^{2} a_{6}^{2} a_{8}^{2} a_{9}-24 a_{11}^{2} a_{6} a_{7} a_{8} a_{9}^{2}+8 a_{11}^{2} a_{7}^{2} a_{9}^{3}\right) .
\end{gathered}
$$

As can be seen from above results, twenty-two classes of quadratic function solutions $f_{i}, 1 \leq i \leq 22$, defined by (8) are constructed. Furthermore, the resulting quadratic function solutions give twenty-two different classes of lump-type solutions $u_{i}, 1 \leq$ $i \leq 22$ to extended $(3+1)$ dimensional NLEE (7). In order to guaranteeing the analyticity of the obtained rational solutions $u_{i}, 1 \leq i \leq 22$, we should choose $a_{11}>0$. All the above rational function solutions $u_{i}, 1 \leq i \leq 22$, go to zero, when the corresponding sum of squares $g^{2}+h^{2} \rightarrow \infty$. As emphasized in the works of 6 2 22 23 24 10], those rational solutions $u_{i}, 1 \leq i \leq 22$, do not approach zero in all directions in $R^{4}$ due to the character of $(3+1)$-dimensions in the resulting solutions. Therefore, those solutions are lump-type solutions but not lump solutions. It is readily seen that the lump-type solutions of (7) corresponding to (11)- 32 are given by

$$
u(x, y, z, t)=-\frac{6\left(a_{1} g+a_{6} h\right)}{f}
$$

where the functions $f, g$ and $h$ are given by Eqs. (8)-10 respectively. In what follows, we present some graphical simulations (Figs.1-7) to better understanding the obtained lump-type solutions. 

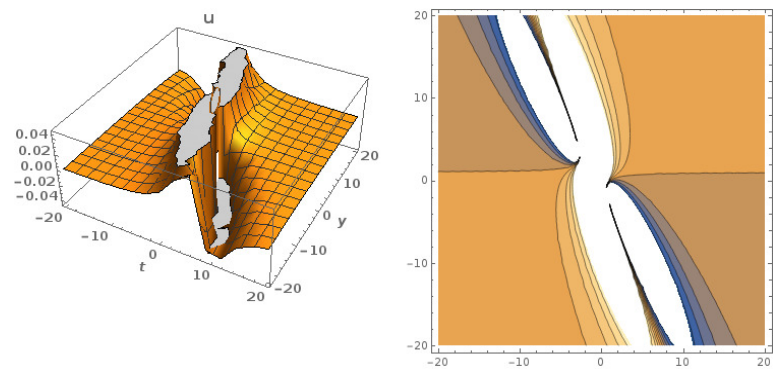

Figure 1. Profiles of the solution (33) with (11) setting all arbitrary parameters to unity except $a_{9}=2$.
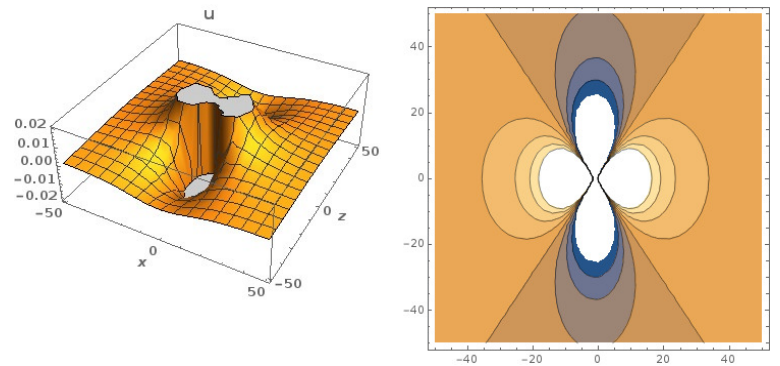

Figure 2. Profiles of the solution (33) with (14) setting all arbitrary parameters to unity
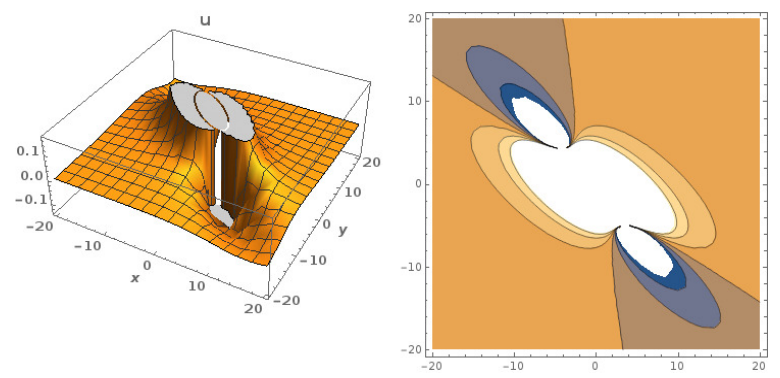

Figure 3. Profiles of the solution (33) with (16) setting all arbitrary parameters to unity except $a_{6}=2$.

\section{Concluding Remarks}

In this work, we have derived a new $(3+1)$ dimesional NLEE through the generalized Hirota bilinear theory. The reason of utilizing this genaralized operators 

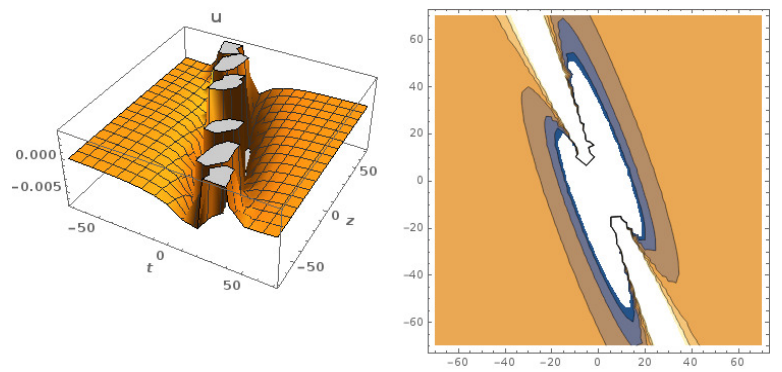

Figure 4. Profiles of the solution (33) with (28) setting all arbitrary parameters to unity except $a_{1}=2$.
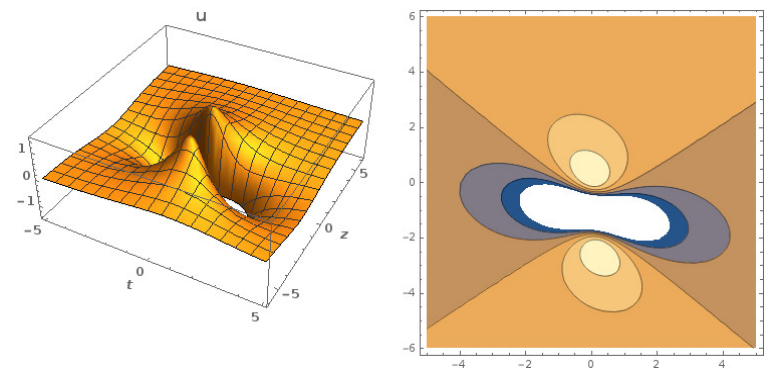

Figure 5. Profiles of the solution (33) with (30) setting all arbitrary parameters to unity except $a_{2}=2$.
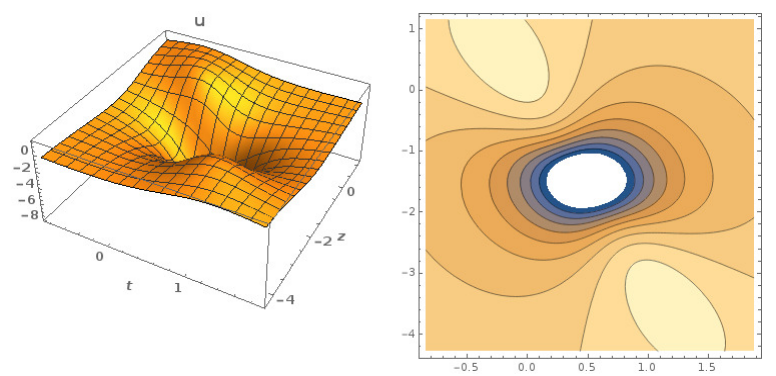

Figure 6. Profiles of the solution (33) with (31) setting all arbitrary parameters to unity.

stems form the shortness and easiness of the obtained Hirota bilinear forms of the extended case. We also note that the deduced extended NLEE is more complicated and contains more nonlinear terms than the classical $(3+1)$ dimesional NLEE. To 

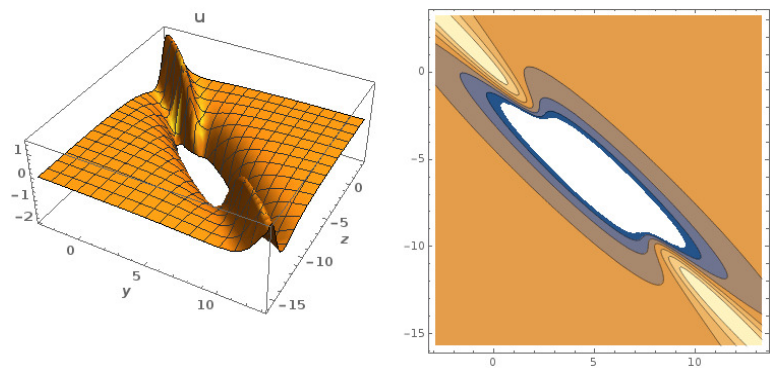

Figure 7. Profiles of the solution (33) with (32) setting all arbitrary parameters to unity.

best of our knowledge, for first time, we have examined a new more complicated $(3+1)$ dimesional equation in the literature. With the help of Maple symbolic computations, twenty-two classes of lump-type solutions are derived from positive quadratic function asssumptions by using the generalized Hirota bilinear form of the extended $(3+1)$-dimensional nonlinear evolution equation. The obtained all rational solutions in this work are lump-type solutions. The analyticity and positivity conditions are satisfied while the rationally condition localized in all directions in $\mathbb{R}^{4}$ is not provided. If one uses the standart Hirota bilinear form (3) over the generalized Hirota bilinear derivatives (6), all the quadratic function solutions presented in this paper are also satisfied for the extended (3+1)-dimensional NLEE. The presented method in this work can be performed to those equations which can be transformed to the Hirota bilinear equations or generalized bilinear Hirota form by some special transformations which are observed from homogenous balance principle.

Authors Contribution Statement Yakup Yıldırım: Visualization, Investigation, Software, Validation, Writing - original draft, Writing -review \& editing. Emrullah Yaşar: Conceptualization, Methodology, Software, Supervision.

Declaration of Competing Interests The authors report no declarations of interest.

Acknowledgements The authors would like to thank the referees for their suggestions and comments.

\section{REFERENCES}

[1] Seadawy, A. R., Iqbal, M., Lu, D., Construction of soliton solutions of the modify unstable nonlinear Schrödinger dynamical equation in fiber optics, Indian J. Phys., 94(6) (2020), 823832. https://doi.org/10.1007/s12648-019-01532-5 
[2] Lü, D. Z., Cui, Y. Y., Lü, C., Huang, S. Y., New interaction solutions of (3+ 1)-dimensional Zakharov-Kuznetsov equation, Indian J. Phys, 87(9) (2013), 897-901. https://doi.org/10.1007/s12648-013-0302-8

[3] Sulaiman, T. A., Bulut, H., Yokus, A., Baskonus, H. M., On the exact and numerical solutions to the coupled Boussinesq equation arising in ocean engineering, Indian J. Phys, 93(5) (2019), 647-656. https://doi.org/10.1007/s12648-018-1322-1

[4] Akram, G., Batool, F., A class of traveling wave solutions for space-time fractional biological population model in mathematical physics, Indian J. Phys, 91(10) (2017), 1145-1148. https://doi.org/10.1007/s12648-017-1007-1

[5] Hirota, R., The direct method in soliton theory, Cambridge University Press, 2004.

[6] Wazwaz, A. M., A variety of distinct kinds of multiple soliton solutions for a $(3+1)$ dimensional nonlinear evolution equation, Math. Methods Appl. Sci., 36(3) (2013), 349-357. https://doi.org/10.1002/mma.2600

[7] Ma, W. X., Lump solutions to the Kadomtsev-Petviashvili equation, Phys. Lett. A, 379(36) (2015), 1975-1978. https://doi.org/10.1016/j.physleta.2015.06.061

[8] Ma, W. X., Qin, Z., Lü, X., Lump solutions to dimensionally reduced p-gKP and p-gBKP equations, Nonlinear Dyn., 84(2) (2016), 923-931. https://doi.org/10.1007/s11071-015-2539-6

[9] Lü, X., Chen, S. T., Ma, W. X., Constructing lump solutions to a generalized Kadomtsev-Petviashvili-Boussinesq equation, Nonlinear Dyn., 86(1) (2016), 523-534. https://doi.org/10.1007/s11071-016-2905-z

[10] Ma, W. X., Lump-type solutions to the (3+1)-dimensional jimbo-miwa equation, Int. J. Nonlinear Sci. Numer. Simul., 17(7-8) (2016), 355-359. https://doi.org/10.1515/ijnsns-20150050

[11] Yang, J. Y., Ma, W. X., Lump solutions to the BKP equation by symbolic computation, Int. J. Mod. Phys. B, 30(28n29) (2016), 1640028. https://doi.org/10.1142/S0217979216400282

[12] Müller, P., Garrett, C., Osborne, A., Rogue waves, Oceanography, 18 (2005), 66-75.

[13] Solli, D. R., Ropers, C., Koonath, P., Jalali, B., Optical rogue waves, Nature, 450 (2007), 1054-1057. https://doi.org/10.1038/nature06402

[14] Yan, X. W., Tian, S. F., Wang, X. B., Zhang, T. T., Solitons to rogue waves transition, lump solutions and interaction solutions for the (3+1)-dimensional generalized B-type KadomtsevPetviashvili equation in fluid dynamics, Int. J. Comput. Math., 96(9) (2019), 1839-1848. https://doi.org/10.1080/00207160.2018.1535708

[15] Geng, X., Ma, Y., N-soliton solution and its Wronskian form of a (3+1)dimensional nonlinear evolution equation, Phys. Lett. A, 369(4) (2007), 285-289. https://doi.org/10.1016/j.physleta.2007.04.099

[16] Wu, J-P., A new Wronskian condition for a $(3+1)$-dimensional nonlinear evolutions equation, Chin. Phys. Lett., 28(5) (2011), 1-3. https://doi.org/10.1088/0256-307X/28/5/050501

[17] Xiao H., Symmetry groups and exact solutions of a $(3+1)$-dimensional nonlinear evolution equation and Maccari's system, Journal of Ningbo University, 24(1) (2011), 108-113.

[18] Ma, W. X., Generalized bilinear differential equations, Stud. Nonlinear Sci., 2 (2011), 140144.

[19] Geng, X., Algebraic-geometrical solutions of some multidimensional nonlinear evolution equations, J. Phys. A Math. Theor., 36(9) (2003), 2289. https://doi.org/10.1088/0305$4470 / 36 / 9 / 307$

[20] Zhaqilao, Rogue waves and rational solutions of a (3+1)-dimensional nonlinear evolution equation, Phys. Lett. A, 377(42) (2013), 3021-3026. https://doi.org/10.1016/j.physleta.2013.09.023

[21] Wazwaz, A. M., New (3+1)-dimensional nonlinear evolution equation: multiple soliton solutions, Cent. Eur. J. Eng., 4(4) (2014), 352-356. https://doi.org/10.2478/s13531-013-0173-y 
[22] Yang, J. Y., Ma, W. X., Abundant lump-type solutions of the Jimbo-Miwa equation in (3+1)-dimensions, Comput. Math. with Appl., 73(2) (2017), 220-225. https://doi.org/10.1016/j.camwa.2016.11.007

[23] Zhang, H. Q., Ma, W. X., Lump solutions to the (2+1)-dimensional Sawada-Kotera equation, Nonlinear Dyn., 87(4) (2017), 2305-2310. https://doi.org/10.1007/s11071-016-3190-6

[24] Yang, J. Y., Ma, W. X., Qin, Z., Lump and lump-soliton solutions to the (2+1)-dimensional Ito equation, Anal. Math. Phys., 8(3) (2018), 427-436. https://doi.org/10.1007/s13324-0170181-9

[25] Yang, J. Y., Ma, W. X., Khalique, C. M., Determining lump solutions for a combined soliton equation in (2+1)-dimensions, Eur. Phys. J. Plus, 135(6) (2020), 494. https://doi.org/10.1140/epjp/s13360-020-00463-z

[26] Ma, W. X., Zhang, Y., Tang, Y., Symbolic computation of lump solutions to a combined equation involving three types of nonlinear terms, East Asian J Appl Math, 10(4) (2020), 732-745. https://doi.org/10.4208/eajam.151019.110420

[27] Ma, W. X., Lump and interaction solutions to linear PDEs in $2+1$ dimensions via symbolic computation, Mod. Phys. Lett. B, 33(36) (2019), 1950457. https://doi.org/10.1142/S0217984919504578

[28] Manafian, J., Lakestani, M., Lump-type solutions and interaction phenomenon to the bidirectional Sawada-Kotera equation, Pramana, 92 (2019), 41. https://doi.org/10.1007/s12043018-1700-4

[29] Manafian, J., Novel solitary wave solutions for the (3+1)-dimensional extended Jimbo-Miwa equations, Comput. Math. with Appl., 76(5) (2018), 1246-1260. https://doi.org/10.1016/j.camwa.2018.06.018

[30] Manafian, J., Mohammadi-Ivatloo, B., Abapour, M., Lump-type solutions and interaction phenomenon to the (2+1)-dimensional Breaking Soliton equation, Appl. Math. Comput., 356 (2019), 13-41. https://doi.org/10.1016/j.amc.2019.03.016

[31] Manafian, J., Mohammed, S. A., Alizadeh, A. A., Baskonus, H. M., Gao, W., Investigating lump and its interaction for the third-order evolution equation arising propagation of long waves over shallow water, Eur. J. Mech. B Fluids, 84 (2020), 289-301. https://doi.org/10.1016/j.euromechflu.2020.04.013

[32] Manafian, J., Lakestani, M., Interaction among a lump, periodic waves, and kink solutions to the fractional generalized CBS-BK equation, Math. Methods Appl. Sci., 44(1) (2021), 1052-1070. https://doi.org/10.1002/mma.6811

[33] Manafian, J., Ilhan, O. A., Avazpour, L., Alizadeh, A. A., N-lump and interaction solutions of localized waves to the $(2+1)$-dimensional asymmetrical Nizhnik-Novikov-Veselov equation arise from a model for an incompressible fluid, Math. Methods Appl. Sci., 43(17) (2020), 99049927. https://doi.org/10.1002/mma.6665 